\title{
The Development of the Newborn Rat Lung in Hyperoxia: A Dose-Response Study of Lung Growth, Maturation, and Changes in Antioxidant Enzyme Activities
}

\author{
JOHN R. BUCHER AND ROBERT J. ROBERTS ${ }^{(41)}$ \\ Division of Neonatology and Clinical Pharmacology, Departments of Pediatrics and Toxicology Center, Department of \\ Pharmacology The University of Iowa, Iowa City, Iowa, USA
}

\section{Summary}

To examine the dose-response relationships of oxygen-induced lung changes, newborn rats were exposed to various patterns of concentrations of hyperoxia $\left(0.4,0.8\right.$, and $\left.>0.95 \mathrm{FiO}_{2}\right)$ for up to 12 days. Prominent findings included microscopic evidence of lung injury and retarded alveolar development (secondary septal development delayed by as much as $88 \%$ ), lower whole lung DNA (50\% of control), lung-to-body-weight ratios (by as much as $18 \%$ ), and significantly less compliance in the lungs after exposures of 6- or 12-day durations to all concentrations of hyperoxia. Significant increases in the activities of the lung protective enzymes superoxide dismutase (129 to $160 \%$ of control), catalase (112 to $274 \%$ of control), and glutathione peroxidase (118 to $256 \%$ of control) were noted when activity was expressed on a DNA basis after 12day exposures to the various patterns of hyperoxia.

Lung changes noted after a 7-day recovery period in air included interstitial thickening ( $117 \%$ of control), persistance of the microscopic injury, and retarded alveolar development seen immediately after initial 6-day hyperoxic exposures. At the conclusion of a second wk of recovery in air, the lungs of hyperoxic exposed animals resembled controls in most respects, but a significantly altered compliance was exhibited by the lungs of animals initially exposed to 6 days of 0.4 or $>0.95 \mathrm{FiO}_{2}$.

The dose dependency of oxygen-induced lung injury is complex. Straightforward, stepwise dose-response adequately describes the evolution of microscopic injury and slowing of alveolar development in hyperoxia, but the dose dependency is not as clearly identified in the oxygen-induced retardation of lung growth including DNA content and in changes in antioxidant enzyme activities. Changes in lung compliance clearly do not follow expected doseresponse relationships.

\section{Speculation}

The toxic influence of oxygen on the lung and on lung development varies depending upon the concentration and duration of oxygen exposure. In addition, individual vulnerability to the toxic effects of oxygen on the lung may vary depending upon the stage of maturation of the lung at the time of oxygen exposure. The variety of clinical presentations and morphologic features of chronic lung disease in premature infants (e.g., Wilson Mikity, chronic pulmonary insufficiency of the premature, and bronchopulmonary dysplasia) may represent a spectrum of oxygen effects on the lung rather than diseases with distinctly different pathogenesis.

Exposure to elevated oxygen concentrations is a commonly used therapy for many conditions including the respiratory distress syndrome of the newborn. Toxic consequences of such therapy in neonates are now recognized in the conditions of retrolental fibroplasia (29) and bronchopulmonary dysplasia (25). Experimental lung injury resulting from exposure to normobaric hyperoxia has been extensively investigated $(2,8,13)$ and found to vary with factors such as age and species $(1,12,38)$. However, the assumption which prevails in clinical medicine, i.e., pulmonary oxygen toxicity increases in a straightforward manner with increases in concentration and duration of hyperoxic therapy, has never been fully investigated in newborn animals (9), although some work has been done in the adult animal $(8,17,28)$. As a consequence, we have explored the character and dose dependency of oxygen-induced lung damage in the newborn rat. These studies include gross, microscopic, and functional and biochemical assessments of the changes in the lungs of newborn rats exposed for up to 12 days to a range of normobaric hyperoxic conditions. Recognizing the possibility that acute insults which appear similar might manifest divergent chronic conditions, studies were also performed after allowing the animals to "recover" in air for 1and 2-wk periods after sublethal exposure.

\section{MATERIALS AND METHODS}

Pregnant rats were obtained from BioLab Corp., St. Paul, MN. Litters born within the 24-hr period before the start of an oxygen exposure were pooled and randomly grouped into litters of 11 pups each. Oxygen exposures were carried out in modified infant incubators (Air Shields, Hatboro, PA) with sufficient flow of the selected gases to provide approximately eight changes of chamber atmosphere per hour. The desired oxygen concentrations were achieved by mixing oxygen with room air. The exposures were conducted under ambient temperature $\left(22-26^{\circ} \mathrm{C}\right)$ with the chamber atmosphere continuously circulated and filtered. Oxygen and carbon dioxide concentrations were periodically monitored with a Beckman Oxygen Analyzer OM-11 and Medical Gas Analyzer LB-2 (Beckman Instruments Inc., Fullerton, CA). Under these exposure conditions $\mathrm{CO}_{2}$ levels did not exceed $0.2 \%$. Chambers were opened daily for approximately $5 \mathrm{~min}$ to provide food (Purina Rat Chow 5001) and water and to rotate the dams between oxygen and air litters.

There were three basic exposure patterns. The first was exposure of newborns to 12 days of $0.21,0.4,0.8$, or $>0.95 \mathrm{FiO}_{2}$ with animals taken for the analyses outlined below on days 1, 3,6, and 12. The second pattern consisted of exposure of newborns to 6 days of 0.4 or $0.8 \mathrm{FiO}_{2}$ followed by 6 days of $>0.95$, with analyses done on days 6 and 12, and comparisons made with air exposed controls. The third pattern included placing animals in $0.21 \mathrm{FiO}_{2}$ for 1 or 2 wk after a 6 -day exposure of the newborns to $0.21,0.4$, 0.8 , or $>0.95 \mathrm{FiO}_{2}$. Dams were rotated among the litters every 48 hr during the "air recovery" type experiments after the pups were 
returned to air. Animals were randomly selected for analysis at the various times which affected a reduction in the litter size to 6 to 8 pups during days 6 to 13 and to 3 to 4 pups for days 14 to 20 .

At the times indicated, animals were weighed and decapitated, the pleural cavity opened, and the lungs were weighed after removal of extrapulmonary tissue. The lungs of animals used in biochemical studies were handled differently in that the heart and lung were removed en bloc, and the lung was perfused through the pulmonary artery with cold isotonic buffer $(0.1 \mathrm{M}$ potassium phosphate; $0.15 \mathrm{M} \mathrm{KCl}$, pH 7.4), and then homogenized for 20 sec with a Polytron (Brinkmann Instruments, Inc., Westbury, NY) at power setting 8 in a 1:10 dilution of the isotonic buffer. DNA analysis of the lung homogenate was done by the method of Richards (32), and the activities of the enzymes superoxide dismutase, EC 1.15.11 (SOD), glutathione peroxidase, EC 1.11.1.9 (GP), and catalase, EC 1.11.1.6 (CAT) were assayed on a 15,000 $\times g$ supernatant of the whole lung homogenate. SOD activity was assayed by the ferricytochrome $c$ method of McCord and Fridovich (22). Glutathione peroxidase activity (using cumene hydroperoxide as substrate) was determined according to the methods of Paglia and Valentine (27), and catalase activity was assessed as described by Holmes and Masters (19).

The lungs of animals used in light and electron microscopic studies were inflated by tracheal infusion of a solution of cacodylate-buffered glutaraldehyde $(3 \%)$ at a pressure of $25 \mathrm{~cm}$ of $\mathrm{H}_{2} \mathrm{O}$. Tracheas were then ligated, and the lungs were immersed in fixative overnight at $4^{\circ} \mathrm{C}$. Two specimens were collected from the middle portion of the left lobe, and one specimen each was collected from the base of the lower lobe and apex of the upper right lobe. The tissues were diced into $1 \mathrm{~mm}$ cubes, postfixed in phosphate-buffered osmium tetroxide $(2 \%)$ for 1 to 2 hours, washed in distilled $\mathrm{H}_{2} \mathrm{O}$, en bloc stained with uranyl acetate, dehydrated in graded methanols, and embedded in Epon 812. Sections of 1 to $2 \mu$ were stained with toluidine blue for light microscopy, and 800 to $1200 \AA$ sections were cut on a LKB Ultratome III for electron microscopy. These sections were then stained in uranyl acetate and lead citrate before examination on a Seimens 101 electron microscope equipped with image intensifier.

Light level morphometric studies were performed on 1 to $2 \mu$ thick sections at a magnification of $\times 1000$ with an AO microscope equiped with a 25 square eyepiece grid. While avoiding large vessels and airways, five random fields per section were selected and line intersections were scored as to their presence over tissue or air space. Additional assessment of lung development was accomplished by determining the number of fully formed and partially completed secondary septa within the grid square at $x$ 400 . Fully formed septa were arbitrarily defined as those structures which exceeded $0.02 \mathrm{~mm}$ in length and protruded from the saccule walls. Partial septa were structures which although similar in appearance and position to fully formed septa, were shorter $(<0.02$ $\mathrm{mm}$ ) and often thicker.

Descending pressure-volume curves were performed in situ on the lungs from animals which were metabolically degassed by oxygen absorption. This was accomplished by the administration of a lethal dose of phenobarbital to rats while they were breathing $100 \%$ oxygen. Using a device similar to that described by Taeusch et al. (35) lungs were inflated with air to a pressure of $30 \mathrm{~cm}$ of $\mathrm{H}_{2} \mathrm{O}$ and allowed to stabilize for $5 \mathrm{~min}$. If the pressure drop during the stabilization period was less than $5 \mathrm{~cm}$ of $\mathrm{H}_{2} \mathrm{O}$, the preparation was accepted for study. The pressure was returned to $30 \mathrm{~cm}$ of $\mathrm{H}_{2} \mathrm{O}$ before being reduced in graded increments at a set rate. Data were collected from the deflation phase only during the initial inflation-deflation cycle (35).

To minimize investigator bias, animals were identified only by a number throughout these procedures. The morphometric and pressure-volume analyses were done in a blind fashion. The animal experiments were performed in accordance with the guidelines of the University of Iowa animal care committee.
RESULTS

Survival, gross appearance, and general activity were similar in the litters exposed to air or the various regimens of hyperoxia with the exception of exposure to 12 days of $>0.95 \mathrm{FiO}_{2}$. In this case, animal activity declined after day 10 , and $27 \%$ of the animals died between day 11 and the end of day 12 . Therefore, the data that are presented for this particular exposure group may be biased in that those animals most susceptible to oxygen-induced injury are not represented.

Table 1 gives information concerning lung and body weights, and the lung to body weight ratios after a 6-day exposure of newborns to $0.21,0.4,0.8$, or $>0.95 \mathrm{FiO}_{2}$ and also after 1 or $2 \mathrm{wk}$ of recovery in air. There were several small but significant differences in the average body weights of animals between the various groups, but no definitive trends were apparent. However, significantly lower lung weights which followed a stepwise dose-response pattern were found at the end of the 6 day exposures in the animals exposed to all three hyperoxic concentrations. The lungto-body weight ratio calculations indicate that hyperoxia produced a selective slowing in the rate of increase in wet lung weight over and above any effect on body weight gain.

During the first wk recovery period, there appeared to be a dose-related rebound in wet lung weight with deficits no longer seen in any group and increases of up to $19 \%$ over control shown by those animals initially exposed to $>0.95 \mathrm{FiO}_{2}$. Lung-to-body weight ratios also reflected this trend with significant elevations in ratio seen in those animals that showed the lowest ratio at the conclusion of the 6-day hyperoxic exposures ( $>0.95$ group). By the end of the second wk in air, the lung-to-body weight ratios had returned to values more appropriate for a 20-day-old rat.

When the 6-day-old animals were continued in hyperoxia for 6 additional days, the deficits in lung weight either remained the same as were seen after the initial 6-day exposure or became larger, depending upon the concentration of hyperoxia employed during the second 6 days (Table 2). Lung-to-body weight ratios were consequently less in the hyperoxic exposed animals than in the air controls. It is apparent, however, that although intraexperimental comparisons of body weight gain showed no effect of hyperoxia (Table 2), comparisons between the experiments (Tables 1 and 2) indicated that animal weight gain in both experimental and control groups was depressed during the 12-day exposures whenever concentrations of oxygen of 0.8 or above were used. This is not a reflection of differences in the initial body weights of the newborn rats before oxygen exposure, as the average newborn weight in each of these experimental groups was approximately $6 \mathrm{~g}$.

The data collected on whole lung DNA content were consistent with the lower wet lung weights in hyperoxic exposed animals. As shown in Figure 1, the DNA content of control rat lungs increased linearly with age. Exposure to the various concentrations of hyperoxia uniformily resulted in a reduced whole lung DNA when expressed as a percentage of experimentally matched control. The patterns do not represent actual decreases in the absolute amount of DNA in the lungs of oxygen exposed animals but rather an impairment of the normal increase associated with lung maturation. Also, there is not a dose or time dependency to the DNA patterns. Although 6- and 12-day exposures to $>0.95 \mathrm{FiO}_{2}$ slowed the increase in lung DNA to the greatest extent (39 and 49\%, respectively), exposure to 12 days of 0.4 and $0.8 \mathrm{FiO}_{2}$ both resulted in a DNA content $29 \%$ less than control. When exposure during the second 6-day period was to $>0.95 \mathrm{FiO}_{2}$ rather than to 0.4 or 0.8 , virtually the same reductions in lung DNA (29 and 34\%, respectively) were observed.

To further assess the extent of oxygen-induced lung injury and to gauge the progress of lung maturation, lungs from the various treatment groups were examined by light and electron microscopy. Exposure to $\mathrm{FiO}_{2} 0.4$ (Fig. $2 \mathrm{~B}$ ) for 6 days produced no discernable changes in the gross microscopic appearance of the lungs of the 
Table 1. Average body weights, lung weights, and lung:body weight ratios after 6 days of hyperoxic exposure and after a 1-or 2-wk recovery period in air

\begin{tabular}{lllllll}
\hline & \multicolumn{2}{c}{ Body wt } & & Lung & & Ratio: lung wt/body wt $^{1}$ \\
$\mathrm{FiO}_{2}$ & $\mathrm{~g}$ & $\%$ of control & $n$ & $\mathrm{~g}$ & $\%$ of control & $n$ \\
\hline
\end{tabular}

6 days of age (entire time in oxygen concentrations indicated)

$\begin{array}{llcll}0.21 & 15.3 \pm 0.3^{2} & & 60 & 0.29 \pm 0.01 \\ 0.4 & 16.2 \pm 0.3 & 106^{3} & 63 & 0.25 \pm 0.01 \\ 0.8 & 15.4 \pm 0.3 & 101 & 65 & 0.25 \pm 0.01 \\ >0.95 & 14.2 \pm 0.4 & 93^{3} & 69 & 0.22 \pm 0.02\end{array}$

13 days of age (first 6 days in respective oxygen concentrations, next 7 days in air

$\begin{array}{ccccccccc}0.21 & 31.6 \pm 0.9 & & 46 & 0.42 \pm 0.03 & & 9 & 1.28 \pm 0.04 & 1.30 \pm 0.04 \\ 0.4 & 28.7 \pm 1.0 & 91^{3} & 59 & 0.37 \pm 0.01 & 88 & 8 & 1.37 \\ 0.8 & 31.0 \pm 0.7 & 98 & 60 & 0.45 \pm 0.02 & 107 & 8 & 1.37 \pm 0.06 & 107 \\ >0.95 & 29.8 \pm 0.8 & 94 & 52 & 0.50 \pm 0.03 & 119 & 8 & 1.63 \pm 0.05 & 127^{4}\end{array}$

20 days of age (first 6 days in respective oxygen concentrations, next 14 days in air)

\begin{tabular}{ccccccccr}
0.21 & $45.5 \pm 1.5$ & & 32 & $0.46 \pm 0.03$ & & 9 & $1.02 \pm 0.05$ & $1.14 \pm 0.08$ \\
0.4 & $46.5 \pm 1.4$ & 102 & 36 & $0.47 \pm 0.03$ & 102 & 8 & 112 \\
0.8 & $52.8 \pm 2.0$ & $116^{4}$ & 35 & $0.59 \pm 0.05$ & $128^{3}$ & 8 & $1.04 \pm 0.09$ & 102 \\
$>0.95$ & $50.1 \pm 2.5$ & 110 & 30 & $0.49 \pm 0.03$ & 106 & 7 & $0.95 \pm 0.04$ & 93 \\
\hline
\end{tabular}

'Lung:body weight ratios were calculated from the individual animals used to obtain lung weight data, thus the $n$ is that given under the lung weight column.

${ }^{2}$ Mean \pm S.E.

${ }^{3} P<0.05$, Student $t$ test.

${ }^{4} P<0.01$, Student $t$ test.

Table 2. Average body and lung weights and lung:body weight ratios after 12 days of exposure to the indicated hyperoxic environments ${ }^{1}$

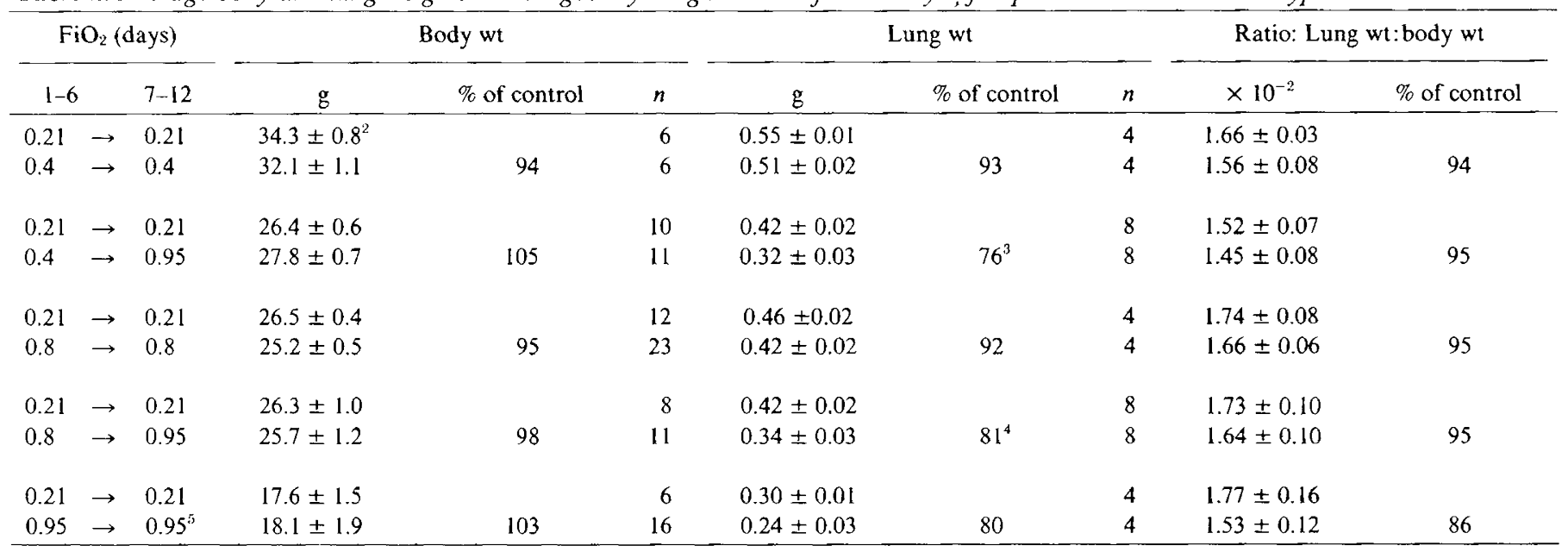

${ }^{1}$ Dams were rotated between air- and oxygen-exposed litters every $24 \mathrm{hr}$.

${ }^{2}$ Mean \pm S.E.

${ }^{3} P<0.01$, Student $t$ test.

${ }^{4} P<0.025$, Student $t$ test.

${ }^{5} 27 \%$ of the animals in this group died by day 12 .

majority of the animals. However, $20 \%$ of those we have examined (3 of 15) showed a marked lack of progress in secondary septal development (Table 3), a process which is normally reaching its maximal activity in the newborn rat lung between the ages of 4 and 13 days (5). By electron microscopic examination, lungs from these affected animals exhibited focal areas of interstitial and perivascular edema, but no evidence of cellular necrosis was noted.

Exposure to 0.8 or $>0.95 \mathrm{FiO}_{2}$ (Fig. 2, C and $D$ ) for 6 days produced a lung which appeared retarded in development in nearly all animals examined (Table 3). In addition, areas of interstitial edema were visible at both the light and electron micrograph levels, and capillaries were engorged. Distended capillaries, found in animals exposed to 0.8 and $>0.95 \mathrm{FiO}_{2}$ for 6 days, have been previously reported in animals exposed to hyperoxia $(2,4)$. Secondary septal development was reduced by $88 \%$ in animals exposed to $>0.95 \mathrm{FiO}_{2}$ for 6 days (Table 3 ), and interstitial edema was found to contribute a large portion of the total saccule wall thickness (Fig. 2D). There was no evidence of an influx of 


\section{Lung DNA Content}
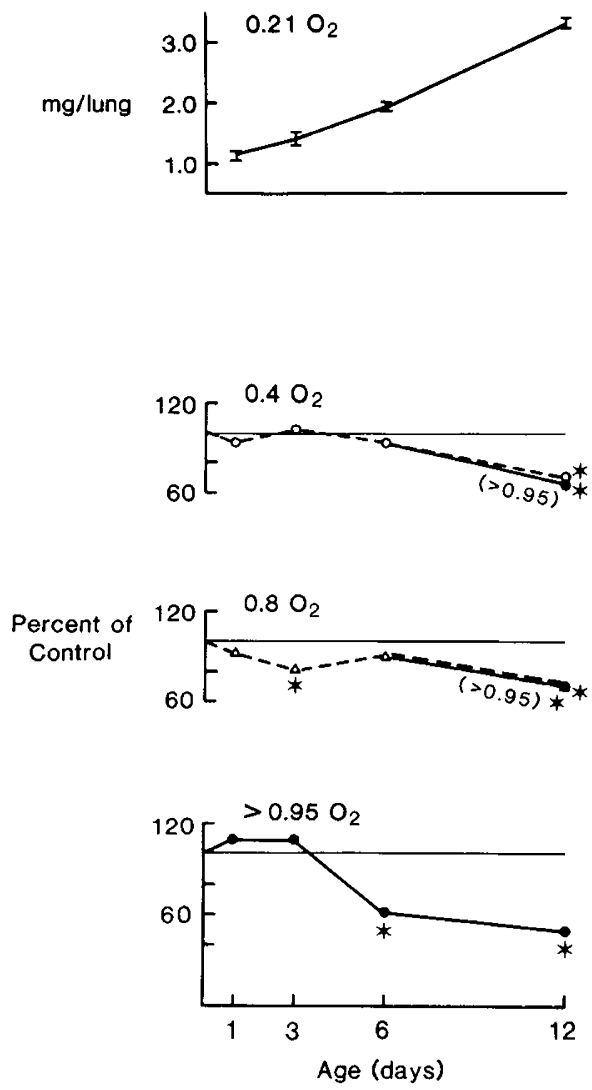

Fig. 1. Top, increase in control lung DNA with age $(n=20$ to 24); bottom, changes in this pattern brought about by exposure to $0.4(O), 0.8$ $(\triangle)$, or $>0.95(\bullet) \mathrm{FiO}_{2}$ during the days indicated, expressed as a percentage of experimentally matched control values $(n=4$ to 12$){ }^{*}, P<0.05$, Feiller's Theorem(10).

pulmonary alveolar macrophages nor of other inflammatory cells, although increased numbers have been observed in the lungs of some animals exposed to $0.8 \mathrm{FiO}_{2}$ or above for 12 days. No evidence of hyaline membranes was found in any of the lungs, but it should be noted that tracheal fixation was used in this study which is not optimal for the preservation of alveolar debri.

Light microscopic examination of the lungs from animals in air recovery experiments demonstrated that the capillary engorgement and interstitial edema induced by the 6-day hyperoxic exposures remained after the first wk in air, but by the end of the second wk the gross appearance of the lungs in all exposure groups was similar. Morphometric estimations of tissue and air space area quantitated the differences between the lung architecture of animals in the various exposure groups (Fig. 3). Rather than the steady reduction in tissue area during days 6 to 20 of age as shown by air exposed control animals, the animals exposed to 6 days of hyperoxia exhibited dose-dependent patterns of change in tissue and air space. Animals initially exposed to 0.8 or greater $\mathrm{FiO}_{2}$ showed an increased lung tissue area during the first week of recovery in air versus the decline shown by animals exposed to 0.21 and $0.4 \mathrm{FiO}_{2}$. Microscopic examination of the lungs of 13day-old animals initially exposed to $>0.95 \mathrm{FiO}_{2}$ revealed that focal areas of edema remained, but that increased numbers of interstitial cells were responsible for the increase in tissue area.

Secondary septal formation progressed in the first wk of recovery, but as shown in Table 3 the dose-related slowing of alveolar development was still clearly evident at this time, and only by the end of the second week in air did it appear that the lungs from hyperoxic exposed animals had achieved a similar stage of alveolar development as the air controls. However, although estimations of secondary septal development are relatively straightforward in animals of 6 and 13 days of age, by the end of the 20th day the lung architecture no longer permits easy estimation of the completeness of alveolar development ty this method because of the similarities in the appearance of alveolar and terminal bronchial walls.

The consequences of exposure of the newborn to hyperoxia can also be seen in physiologic measurements of lung function. Both the maximal lung volume at $30 \mathrm{~cm}$ of $\mathrm{H}_{2} \mathrm{O}$ pressure (Table 4) and the compliance characteristics (Fig. 4) of the lungs of hyperoxic exposed animals appeared altered. Although not significant, the hyperoxic exposed animals showed a tendency toward lower lung volumes both immediately after the 6-day exposures and after 1 wk of recovery in air. This difference has been masked in the deflation pressure volume curves of Figure 4 by normalizing the lung volumes to percentage of maximal lung volume at $30 \mathrm{~cm}$ of pressure. Nonetheless, significantly less compliance was seen in the lungs from 6-day-old animals in 0.4 and $>0.95 \mathrm{FiO}_{2}$ exposure groups when compared to controls. Significant differences were also present in the lungs from 20-day-old animals initially exposed to 6 days of 0.4 or $>0.95 \mathrm{FiO}_{2}$ in their compliance properties at 30 to 80 and 60 to $90 \%$ of maximal lung volume, respectively. Lung compliance was not significantly altered from control in all the 1 wk recovery animals and in the animals exposed to $0.8 \mathrm{FiO}_{2}$ at all times tested.

The lung enzyme activities of SOD, GP, and CAT were assayed after $1,3,6$, or 12 days of exposure to the various hyperoxic regimens. In Figures 5, 6, and 7, the enzyme activities in the lungs of control and experimental animals are presented. The enzyme activities for controls are expressed on the basis of whole lung and per mg DNA. Changes in these enzyme activities brought about by exposure of the animals to the various hyperoxic regimens are expressed as a percentage of experimentally matched control.

Control whole lung SOD activity (Fig. 5) increased with age after a relatively stable period from days 1 to 3 . When SOD activity was expressed per mg DNA, the values remained essentially unchanged. Exposure to the various hyperoxic conditions resulted in activities either similar to control or significantly lower after 6 and 12 days of $>0.95 \mathrm{FiO}_{2}$ when data were expressed per whole lung. When SOD activities in the lungs of hyperoxic exposed animals were expressed on a per mg DNA basis, significant increases were seen after 6 days of exposure to $>0.95 \mathrm{FiO}_{2}$ and after 12 days exposure to all hyperoxic regimens.

Whole lung GP activity (Fig. 6) declined in the control animals from days 1 to 6 and regained the level found in the 1 day animals by day 12 . A decrease in control GP activity per cell over the first 12 days is suggested by the decline in activity when expressed per mg DNA. Exposure to the hyperoxic regimens brought about no significant changes in the whole lung GP activities after exposures of $1,3,6$, or 12 days. When enzyme activities were expressed per mg DNA, increases similar to those noted in lung SOD activity were seen.

Figure 7 presents data on lung CAT activities. Whole lung CAT activity of control animals increased with age, but declined on a per mg DNA basis. Again, when expressed on a whole lung basis, with one exception there were no changes in lung CAT activity brought about by the hyperoxic exposures. Increases were noted when CAT activity was expressed per mg DNA.

\section{DISCUSSION}

Since the early 1930's, it has been recognized that immature animals of certain species are better able to tolerate exposure to hyperoxia than are adult animals (33). That this resistance to oxygen-induced lung injury is only relative and not absolute has been demonstrated in the newborn mouse $(4,18,21,30)$, pig (39), guinea pig (26), and rat (9). Descriptions of microscopic and biochemical lung changes have been reported after exposures of 


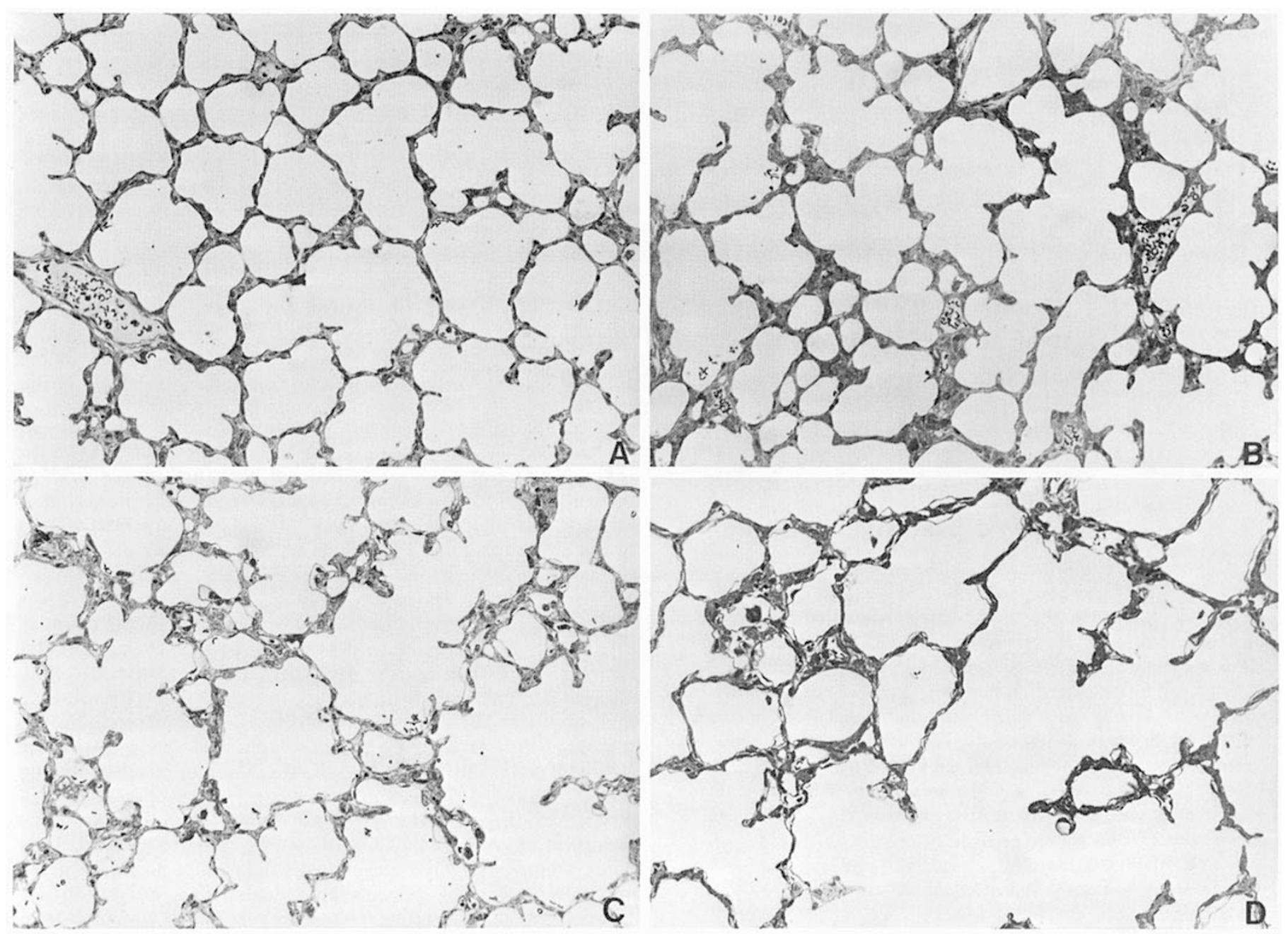

Fig. 2. Photomicrographs of lungs from newborn rats after exposure to $\mathrm{FiO}_{2}, 0.21 ; B, 0.4 ; C, 0.8$; or $D$, >0.95 for 6 days. Magnification, $\times 46$.

newborn and weanling animals of various species to concentrations of hyperoxia ranging from 0.4 to $1.0 \mathrm{FiO}_{2}(7,21)$. What has been lacking, however, is a single study of quantitative comparisons of structural, functional, and biochemical changes in the lungs of animals during and after exposure to various oxygen concentrations. This study was designed to examine changes in a number of different indices of lung morphology, biochemistry, and physiology to determine their dose and time dependency in the newborn rat.

The results of these studies indicate that the dose-response relationship between exposure of the newborn to hyperoxia and consequent lung injury is complex, with the degree of dose dependency differing with the particular injury parameter studied. Complicating the understanding of these relationships is the observation that hyperoxic exposure of the newborn results in a profound inhibition in the devleopment of the lung. This aspect of the potential hazards associated with hyperoxic therapy in the newborn has not received the attention accorded the more classically recognized spectrum of oxygen-induced lung damage.

There are several potential mechanisms which may play a role in the oxygen effect on lung growth and maturation. Several investigators have reported an inhibition in the synthesis of DNA in the lungs of newborn animals exposed to $>0.95 \mathrm{FiO}_{2}(9,23$, 24). These observations compliment other results concerning the in vitro inhibition of growth of several different nonpulmonary cell lines under concentrations of hyperoxia as lcw as $30 \%(6,31)$. In addition to this apparently direct mechanism of action on cell growth is the poissibility that lung growth in hyperoxia may be influenced indirectly through an adaptive response which acts to balance the diffusion capacity of the lung to the concentration of oxygen in the environment. Burri and Weibel (7) and Bartlet (3) have reported morphologic studies in which exposure of weanling animals to 2 to $3 \mathrm{wk}$ of various concentrations of hypoxia and hyperoxia resulted in compensatory changes in several parameters related to lung growth and alveolar surface area. Hyperoxia brought about a slowing of lung maturation and a reduction in alveolar number and surface area, whereas hypoxia led to increases in pulmonary diffusion capacity. A third mechanism with the potential to influence the rate and pattern of lung growth is the direct toxic action of hyperoxia on the cells of the lung, resulting in the destruction of the capillary endothelium and alveolar epitheliunit. This has been reported by many investigators and reviewed by Weibel (37). All three mechanisms have the potential to affect the growth and development of the lung in hyperoxia. The results presented herein along with our previous reports $(1,38)$ indicate that the contribution of each may well depend on the particular concentration and duration of hyperoxic exposure as well as on the age of the animal at the time of exposure.

Several investigators have proposed that a major portion of the observed effect of hyperoxia on DNA synthesis might result from inequities in control and experimental animal nutrition. In the reports cited above $(9,24)$, the gains in body weight of oxygenexposed animals were less than those of controls. Hackney et al. (16) have concluded from experiments with fed and semistarved mice exposed to hyperoxia that food deprivation would contribute 
Table 3. Effect of hyperoxia on lung development

\begin{tabular}{llll}
\hline \multicolumn{1}{c}{$\mathrm{FiO}_{2}$ group } & $n^{1}$ & Septa $^{2}$ & Partial sep \\
\hline 6 days $^{3}$ & & & \\
& & & \\
0.21 & 6 & $2.7 \pm 0.5^{4}$ & $7.3 \pm 0.6$ \\
0.4 & 6 & $2.6 \pm 0.3$ & $5.1 \pm 0.6^{5}$ \\
0.8 & 6 & $1.5 \pm 0.3$ & $5.3 \pm 0.5^{5}$ \\
$>0.95$ & 4 & $0.3 \pm 0.1^{5}$ & $1.8 \pm 0.7^{5}$
\end{tabular}

13 days $^{3}$

$\begin{array}{llll}0.21 & 3 & 5.6 \pm 0.4 & 8.9 \pm 2.3 \\ 0.4 & 3 & 4.5 \pm 0.6 & 6.3 \pm 0.8 \\ 0.8 & 3 & 3.7 \pm 0.6 & 9.3 \pm 1.4 \\ >0.95 & 3 & 3.1 \pm 0.7^{5} & 6.5 \pm 2.4\end{array}$

20 days $^{3}$

$\begin{array}{llll}0.21 & 3 & 2.2 \pm 0.3 & 6.1 \pm 0.3 \\ 0.4 & 3 & 2.0 \pm 0.3 & 4.9 \pm 0.3^{5} \\ 0.8 & 3 & 2.0 \pm 0.4 & 4.2 \pm 0.8 \\ >0.95 & 5 & 2.1 \pm 0.5 & 3.9 \pm 0.8\end{array}$

${ }^{1} n=$ number of lungs examined in each of the treatment groups.

${ }^{2}$ Progress of secondary septal formation was ascertained by septal counts as described in "Materials and Methods," with the exception of those septal numbers derived from the lungs of 20 day animals. These were estimated by the number of thin septa intersecting a $5 \mathrm{~mm}$ line in 10 fields at $\times 400$, a technique which was better suited for counting fully formed septa in lungs at this stage of development. Partial septa were counted by the same method used for days 6 and 13 .

${ }^{3}$ Exposure during days 1 to 6 was in the concentrations of oxygen indicated under $\mathrm{FiO}_{2}$ group. Thereafter, all groups were exposed to air for 1 (13 days) or 2 wk (20 days).

${ }^{4}$ Mean \pm S.E.

${ }^{5}$ Significantly different from control, Student $t$ test $P<0.025$.

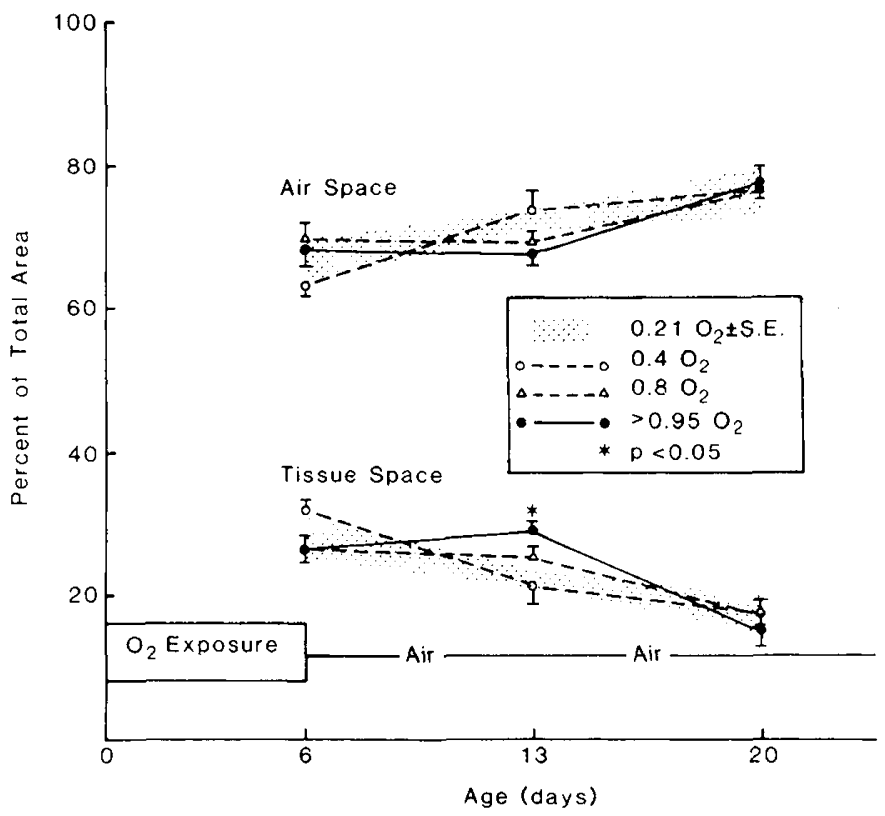

Fig. 3. Morphometric estimations of tissue space and air space in the lungs from animals exposed to hyperoxia or air for 6 days and after 7 and 14 days of recovery in air. Values (means $\pm S$.E. for three to six animals) representing the percentage of total area derived by the point count technique described in "Materials and Methods" are plotted versus animal age in days, ${ }^{*}, P<0.05$, Feiller's Theorem (10).
Table 4. Maximum lung volume at $30 \mathrm{~cm}$ of $\mathrm{H}_{2} \mathrm{O}$ pressure $\left(M L V_{30}\right)$ in lungs from animals in the various exposure groups

\begin{tabular}{ccrrrrr}
\hline $\begin{array}{c}\mathrm{FiO}_{2} \\
\text { group }\end{array}$ & 6 days $^{1}$ & $n$ & 13 days $^{1}$ & $n$ & 20 days $^{1}$ & $n$ \\
\hline 0.21 & $0.96 \pm 0.04^{2}$ & 9 & $1.41 \pm 0.05$ & 10 & $2.88 \pm 0.09$ & 16 \\
0.4 & $0.90 \pm 0.03$ & 9 & $1.25 \pm 0.04$ & 10 & $2.92 \pm 0.13$ & 13 \\
0.8 & $0.92 \pm 0.05$ & 10 & $1.26 \pm 0.07$ & 9 & $2.96 \pm 0.11$ & 13 \\
$>0.95$ & $0.88 \pm 0.03$ & 18 & $1.29 \pm 0.06$ & 18 & $2.63 \pm 0.14$ & 20 \\
\hline
\end{tabular}

${ }^{1}$ Exposure during days 1 to 6 was in the concentrations of oxygen indicated under $\mathrm{FiO}_{2}$ group. Thereafter, all groups were exposed to air for 1 (13 days) or 2 wk ( 20 days).

${ }^{2}$ Means \pm S.E. There are no statistically significant differences in these data within each age group.

significantly to the oxygen-induced inhibition of DNA synthesis. To our knowledge, the data reported herein are the first which indicate significant effects of oxygen exposure in slowing the increase in lung DNA in animals whose weight gains were similar to those of controls. However, an interexperimental comparison of the body weights in Table 2 shows a dose-related reduction of the body weights of the air exposed as well as of the oxygen exposed animals after 12-day hyperoxic exposures. In these experiments, dams were rotated between oxygen and air control litters every $24 \mathrm{hr}$. The equivalent weight gains in the control and hyperoxic exposed groups suggest that the dams were sufficiently affected by oxygen exposures of 0.8 or greater $\mathrm{FiO}_{2}$ to influence their ability to normally nurture the pups. As a consequence, we cannot entirely exclude the possibility that inadequate nutrition is a corequsite for the oxygen-induced inhibition of increases in lung DNA.

Microscopic lung injury was assessed in animals after 6-day exposures in $0.21,0.4,0.8$, and $>0.95 \mathrm{FiO}_{2}$. In general, lung damage appeared dose dependent and similar to descriptions previously published (21). Although the deficits in lung secondary septal development were most obvious in lungs which showed microscopic injury ( $\mathrm{FiO}_{2}, 0.8$ and $>0.95$ groups), we have no evidence which would lead us to conclude that the two phenomena are related in terms of cause and effect. However, $20 \%$ of the animals examined after a 6 -day exposure to $0.4 \mathrm{FiO}_{2}$ showed deficits in secondary septal formation independent of the development of capillary engorgement or the formation of extensive interstitial edema. This variability in response to $0.4 \mathrm{FiO}_{2}$ in terms of slowed septal development may represent differences in the susceptibility of individual animals to oxygen-induced lung injury which may not be as apparent after exposure to the higher oxygen concentrations.

Differences in individual animal susceptibility are not adequate to explain the pattern of oxygen-induced changes in lung compliance. Decreased lung compliance was shown in the lungs from animals exposed to 0.4 or $>0.95 \mathrm{FiO}_{2}$ both immediately after a 6day exposure and again after a 2 -wk recovery period in air. In contrast, those animals exposed to $0.8 \mathrm{FiO}_{2}$ for 6 days maintained compliance characteristics identical to control at all test times. We have no explanation for the lack of apparent effect of exposure to $0.8 \mathrm{FiO}_{2}$ on lung compliance. In many respects, the lungs from animals in this exposure group closely resembled the lungs of animals exposed to $>0.95 \mathrm{FiO}_{2}$, and appeared to at least qualitatively possess the same patterns of injury. Although interstitial edema and capillary engorgement are known to have an effect on pulmonary compliance characteristics (20), the results of this study point out the need for an investigation of possible dose-related, oxygen-induced changes in newborn lung surfactant. The need to examine the dose-dependent effects of hyperoxia on pulmonary surfactant in the newborn is further emphasized by the conflicting reports in the literature in studies of adults of several species $(15$, 36).

In recent years, evidence has accumulated which favors a 
A. $\mathrm{O}_{2}$ EXPOSURE

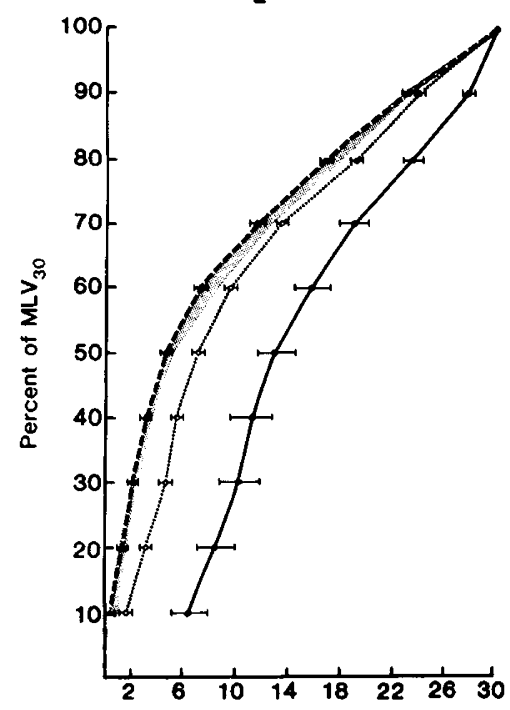

B. RECOVERY

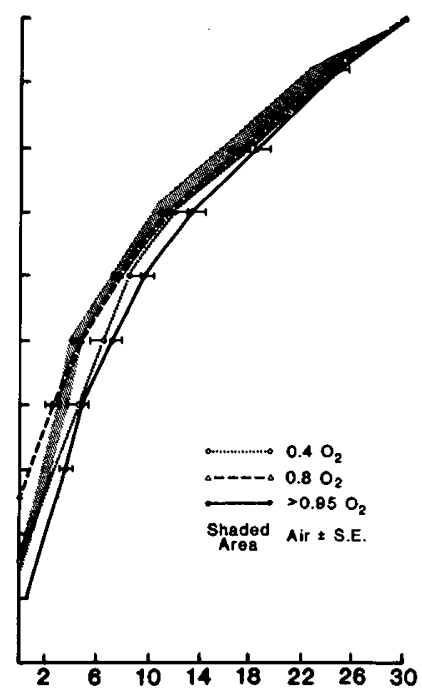

C. RECOVERY

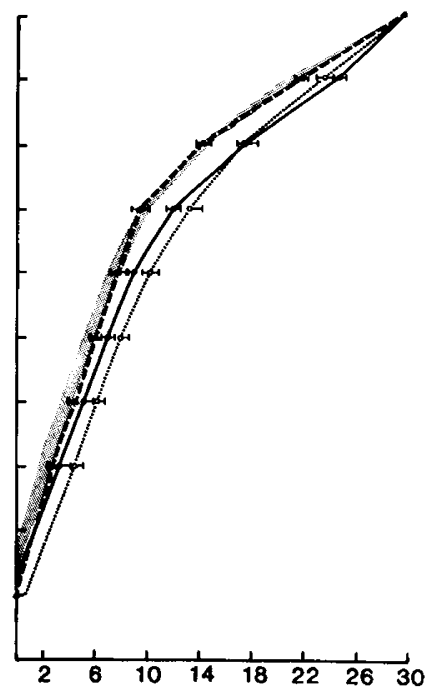

Pressure $\left(\mathrm{cm} \mathrm{H}_{2} \mathrm{O}\right)$

Fig. 4. Descending pressure-volume curves (means \pm S.E. of eight to 14 animals) were generated after 6-day exposures to air or hyperoxia $(A)$ and after 7- $(B)$ and 14- $(C)$ day air recovery periods. Significant differences from air control were found in the $0.4 \mathrm{FiO}_{2}$ exposed 6-day-old animals from 20 to $60 \%$ of $\mathrm{MLV}_{30}$, and in $>0.95 \mathrm{FiO}_{2}$ exposed 6-day animals from 20 to $90 \%$ of $\mathrm{MLV}_{30}$. Significant differences were also found in $0.4 \mathrm{FiO} \mathrm{F}_{2}$ exposed 20-day-old animals at 30 to $80 \%$ of $\mathrm{MLV}_{30}$ and also in $>0.95 \mathrm{FiO}_{2}$ exposed 20-day-old animals at 60 to $90 \%$ of $\mathrm{MLV}_{30}(P<0.01$, Student's $t$ test $)$.

\section{Superoxide Dismutase}
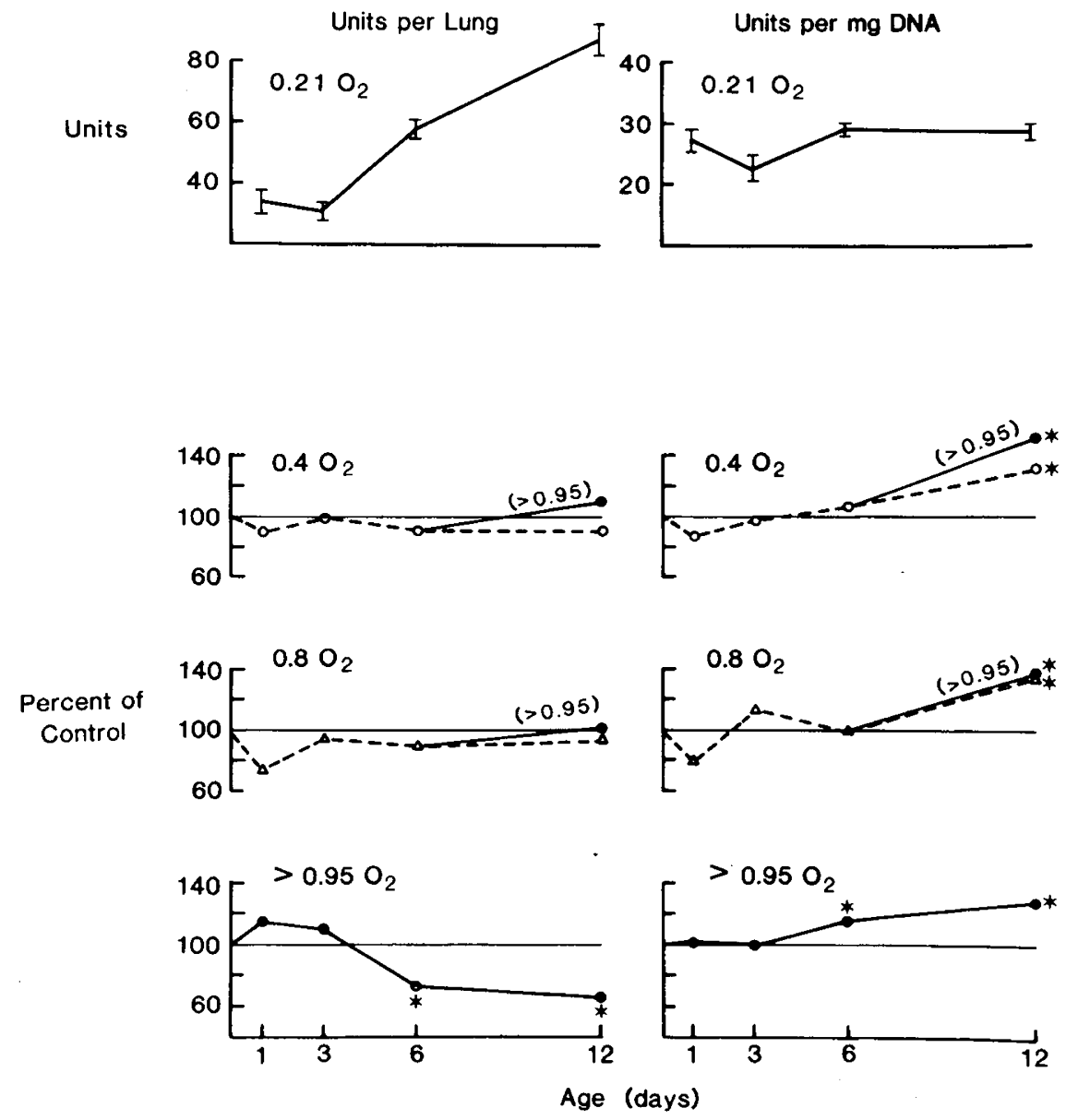

Fig. 5. The activity of lung superoxide dismutase expressed as units per whole lung (left) and units per mg DNA (right). Control lung SOD activities in 1- to 12-day-old rats are shown in the top panel $(n=20$ to 24), and changes in these activities are shown in the lower panels after exposures to 0.4 $(\bigcirc), 0.8(\triangle)$, or $>0.95(\bullet) \mathrm{FiO}_{2}(n=4$ to 12$)$, with values expressed as a percentage of experimentally matched control. ${ }^{*}, P<0.05$, Feiller's Theorem (10). 
protective role for the lung antioxidant enzymes SOD, GP, and CAT against oxygen-induced lung injury $(1,11,12,14,34,38)$. An association has been described between the longer duration of survival in hyperoxia and the ability of neonatal animals of certain species including the rat to rapidly increase the activities of these enzymes after hyperoxic challenge (12). To further explore the relationship between the lung antioxidant enzyme activities and the patterns of lung injury described in this report, we have assayed the activities of lung SOD, GP, and CAT throughout the various exposures. Unfortunately, the usual bases for expression of the enzyme activity data (i.e., DNA and whole lung) also are influenced by prolonged hyperoxic exposure. Enzyme activities based on protein content or per gram lung weight are subject to influence by the presence of interstitial edema. Alternatively, expression of activity per whole lung and per mg DNA as done in this report will be influenced when comparisons are made between groups of animals with different rates of lung growth. Recognizing these problems and also recognizing that the enzymes are not uniformily distributed between the various cell types of the lung (34) and therefore will be influenced by changes in cell populations, some limited conclusions can be drawn. Significant increases in enzyme activity are seen in the lungs of animals beyond 6 days of hyperoxia when data are expressed per mg DNA, but a doseresponse relationship is not obvious. Enzyme activities expressed on a whole lung basis show no significant increases with one exception. If the changes in enzyme activity expressed per $\mathrm{mg}$ DNA represent biologically significant events for certain cell types in the lung, these increases are consistent with previous reports dealing with the relative resistance of the neonatal rat to oxygeninduced lethality $(1,34,38)$. However, the increases in enzyme activity are occurring concurrent with the evolution of microscopic lung injury, deterioration of pressure volume relationships, and concurrent with the observed inhibition of lung growth and development. Although these findings reconfirm the association between increases in lung antioxidant enzyme activities and survival in hyperoxia, we have been unable to identify an association between any dose-related change or lack of change in enzyme activity and the extent of pulmonary damage as defined by any one particular injury parameter. Further studies are needed to delineate the role of the protective enzymes in the individual biochemical events which lead to direct lung injury and/or disruption of pulmonary maturation.

In summary, exposure of the newborn to concentrations of hyperoxia as low as $0.4 \mathrm{FiO}_{2}$ results in significant impairment of lung growth and maturation, changes the compliance characteristics of the lung, and results in microscopic evidence of injury. The dose dependency of these events differs and does not always follow a stepwise pattern. Interpretations of changes in lung antioxidant enzyme activities in prolonged hyperoxia are complicated by the influences of hyperoxic exposure on lung DNA content and lung growth. If these studies are directly applicable to the human infant, many of the clinical changes noted in infants receiving prolonged hyperoxic therapy may be the result of oxygen-induced alterations in lung development. Variable rates and

\section{Glutathione Peroxidase}
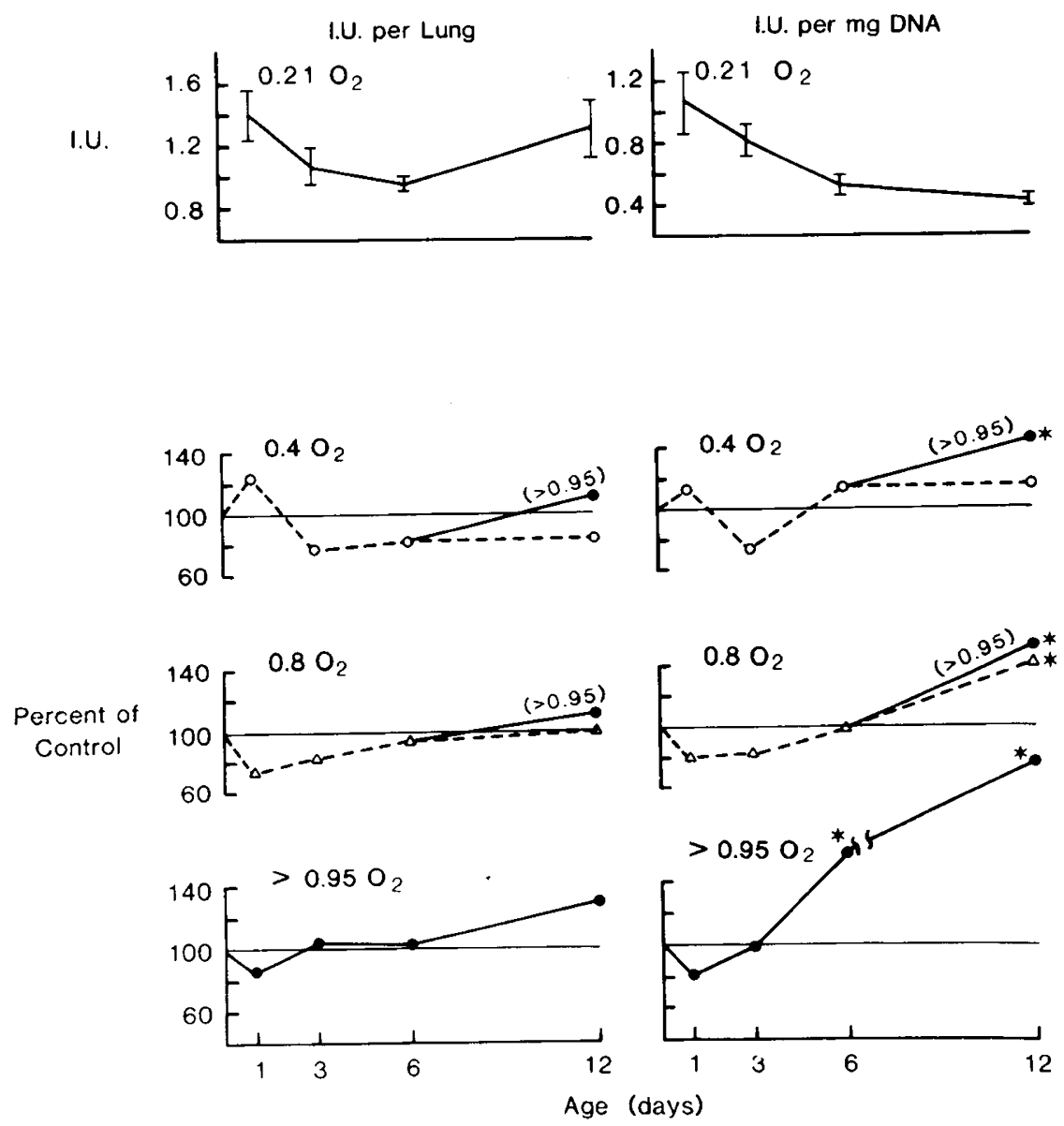

Fig. 6. The activity of lung glutathione peroxidase expressed as international units per whole lung (left) and per mg DNA (right). Control lung GP activities in 1- to 12-day-old rats are shown in the top panel $(n=20$ to 24), and changes in these activities are shown in the lower panels after exposures to $0.4(\bigcirc), 0.8(\triangle)$ or $>0.95(\bullet) \mathrm{FiO}_{2}(n=4$ to 12$)$, with values expressed as a percentage of experimentally matched control. *, $P<0.05$, Feiller's Theorem (10). - --, value for GP activity per mg DNA of $256 \%$ of control in the 12-day $>0.95 \mathrm{FiO}_{2}$ group. 


\section{Catalase}

\section{I.U. per Lung I.U. per mg DNA}
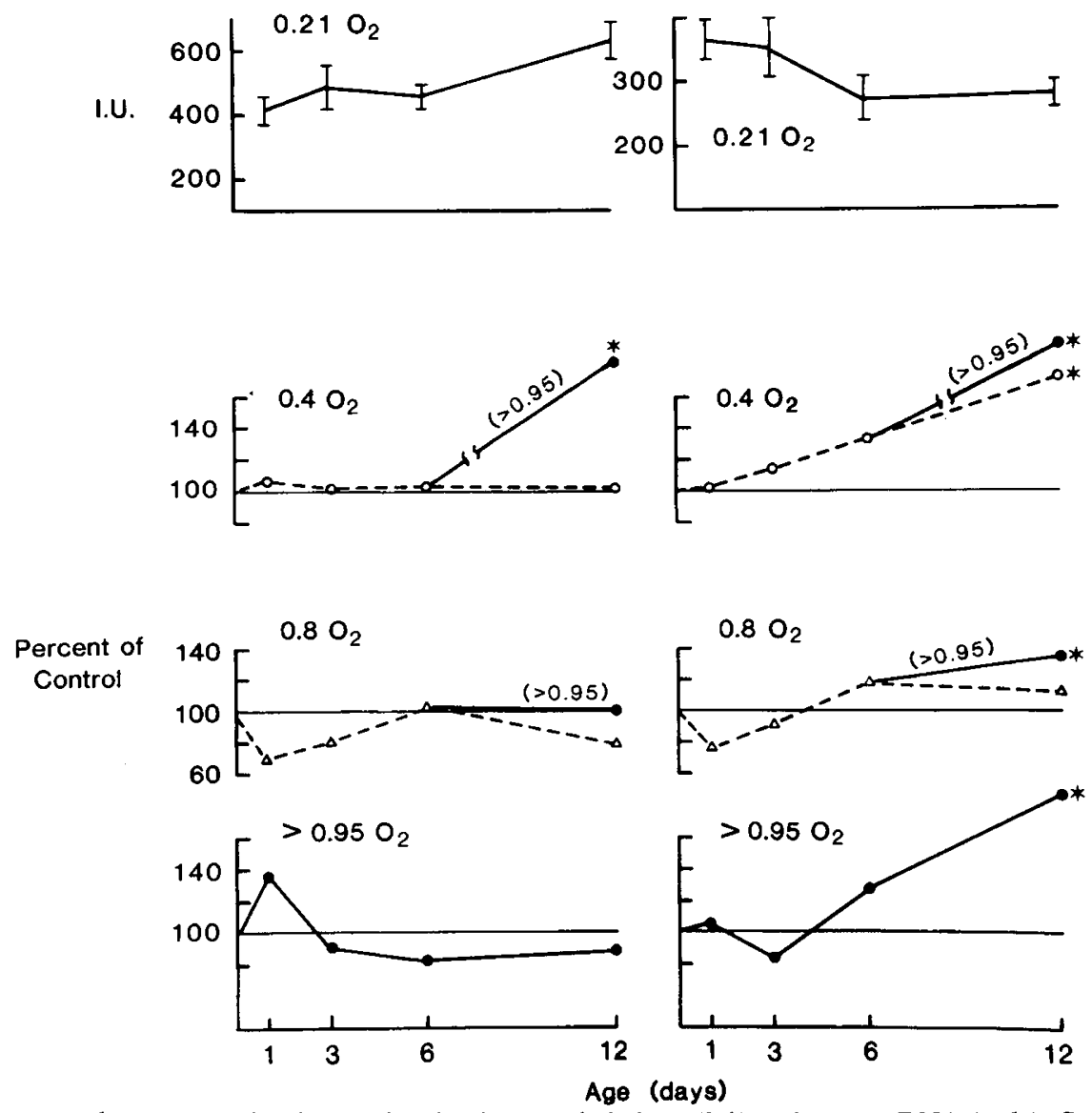

Fig. 7. The activity of lung catalase expressed as international units per whole lung (left) and per mg DNA (right). Control lung CAT activities in 1- to 12-day-old rats are shown in the top panel $(n=20$ to 24), and changes in these activities are shown in the lower panels after exposures to $0.4(O)$, $0.8(\triangle)$ or $>0.95(\bullet) \mathrm{FiO}_{2}(n=4$ to 12$)$, with values expressed as a percentage of experimentally matched control. ${ }^{*}, P<0.05$, Feiller's Theorem (10). ----, values off the scale of the graph. Twelve-day-old rats exposed for 6 days to $0.4 \mathrm{FiO}_{2}$ and continued for 6 additional days in $>0.95 \mathrm{FiO}_{2}$ gave $\mathrm{CAT}$ activities 203 and $274 \%$ of control when expressed per lung and per mg DNA, respectively.

degrees of completion of normal maturatiion processes might be anticipated both during hyperoxic exposure and after the infant is returned to normoxia. Thus, accurate assessment of the involvement of oxygen in the various forms of chronic lung disease in premature infants, such as bronchopulmonary dysplasia, would require appreciation of these facts. The results of this study coupled with appropriate epidemiologic efforts recognizing the importance of lung changes produced by exposure to relatively low levels of hyperoxia, should provide a better understanding of the ramifications of oxygen therapy in the newborn particularly in its role in the development of bronchopulmonary dysplasia and other chronic lung states.

\section{REFERENCES AND NOTES}

1. Autor, A. P., Frank, L., and Roberts, R. J.: Developmental characteristics of pulmonary superoxide dismutase: relationship to idiopathic respiratory distress syndrome. Pediatr. Res., 10: 154 (1976).

2. Balentine, J. D.: Experimental pathology of oxygen toxicity. In: F. F. Jobsis: Oxygen and Physiological Function. pp. 311-378 (Professional Information Library, Dallas, 1977).

3. Bartlett, D. Jr.: Postnatal growth of the mammalian lung: influence of low and high oxygen tensions. Respir. Physiol., 9: 58 (1970).

4. Bonikos, D. S., Bensch, K. G., Ludwin, S. K., and Northway, W. H.: Oxygen toxicity in the newborn: the effect of prolonged 100 percent $\mathrm{O}_{2}$ exposure on the lungs of newborn mice. Am. J. Pathol., 85: 623 (1976).

5. Brody, J. S., and Vaccaro, C.: Postnatal formation of alveoli: interstitial events and physiologic consequences. Fed. Proc., 38: 215 (1979).

6. Broesmer, R. W., and Rutter, W. J.: The effect of oxygen tension on the growth and metabolism of a mammalian cell line. Exp. Cell Res., 25: 101 (1961).
7. Burri, P. H., and Weibel, E. R.: Morphometric estimation of pulmonary diffusion capacity. II. Effect of $\mathrm{PO}_{2}$ on the growing rat lung. Respir. Physiol., 11: 247 (1971).

8. Clark, J. M., and Lambertsen, C. J.: Pulmonary oxygen toxicity. A review. Pharmacol. Rev., 23: 37 (1971).

9. Craig, J., Rev-Kury, L. H., and Kury, G.: Oxygen toxicity in newborn rats. Lab. Invest. (Abstract), 31: 425 (1974).

10. Finney, D. J.: Statistical Method in Biological Assay. pp. 26-35 (Charles Griffin and Co. Ltd., London, 1971).

11. Frank, L., Autor, A. P., and Roberts, R. J.: Oxygen therapy and hyaline membrane disease: the effect of hyperoxia on pulmonary superoxide dismutase and the mediating role of plasma or serum. J. Pediatr., 90: 105 (1977).

12. Frank, L., Bucher, J. R., and Roberts, R. J.: Oxygen toxicity in neonatal and adult animals of various species. J. Appl. Physiol., 45: 699 (1978).

13. Frank, L., and Massaro, D.: The lung and oxygen toxicity. Arch. Intern. Med., 139: 347 (1979).

14. Frank, L., Yam, J., and Roberts, R. J.: The lung of endotoxin in protection of adult rats from oxygen-induced lung toxicity. J. Clin. Invest., 61: 269 (1978).

15. Fujiwara, T., Adams, F. H., and Seto, K.: Lipids and surface tension of extracts of normal and oxygen-treated guinea pigs. J. Pediatr., 65: 45 (1964).

16. Hackney, J. D., Evans, M. J., Bils, R. F., Spier, C. E., and Jones, M. P.: Effects of oxygen at high concentrations and food deprivation on cell division in lung alveoli of mice. Exp. Mol. Pathol., 26: 350 (1977).

17. Hayatdavoudi, G., Crapo, J. D., Foscue, H. A., and O'Neil, J.: Evidence of toxicity of 60\% oxygen on rat lungs. Am. Rev. Respir. Dis., 177: 346 (1978).

18. Hellstrom, B., and Nergardh, A.: The effect of high oxygen concentrations and hypothermia on the lung of the newborn mouse. Acta Paediatr. Scand., 54: 457 (1965).

19. Holmes, R. S., and Masters, C. J.: Epigenetic interconversions of the multiple forms of mouse liver catalase. FEBS Lett., 11: 45 (1970).

20. Hoppin, F. G., Jr., and Hilderbrandt, J.: Mechanical properties of the lung. In: J. B. West: Bioengineering Aspects of the Lung. pp. 83-162 (Marcel Dekker, New York, 1977). 
21. Ludwin, S. K., Northway, W. H., and Bensch, K. G.: Oxygen toxicity in the newborn: necrotizing bronchiolitis in mice exposed to 100 percent oxygen. Lab. Invest., 31: 425 (1974).

22. McCord, J. M. and Fridovich, I.: Superoxide dismutase: an enzymatic function for erythrocuprein (Hemocuprein). J. Biol. Chem., 244: 6049 (1969).

23. Northway, W. H.. Petriceks, R., and Shahinian, L.: Quantitative aspects of oxygen toxicity in the newborn: inhibition of lung DNA synthesis in the mouse. Pediatrics, 50: 67 (1972).

24. Northway, W. H., Rezeau, L., Petriceks, R., and Bensch, K. G.: Oxygen toxicity in newborn lung: reversal of inhibition of DNA synthesis in mouse. Pediatrics, 57: 41 (1976)

25. Northway, W. H., Rosan, R. C., and Porter, D. Y.: Pulmonary disease following respiratory therapy of hyaline-membrane disease: bronchopulmonary dysplasia. N. Engl. J. Med.. 276: 357 (1967).

26. Northway, W. H., Rosan, R. C., Shahinian, L., Castellio, R. A., Gyepes, M. T. and Durbridge, T.: Radiologic and histologic investigation of pulmonary oxygen toxicity in newborn guinea pigs. Invest. Radiol. 4: 148 (1969)

27. Paglia. D. E., and Valentine, W. N.: Studies on the quantitative and qualitative characterization erythrocyte glutathione peroxidases. J. Lab. Clin. Med., 70: 158 (1967).

28. Pariente, P. R., LeGrande, M., and Brouet, G.: Aspects ultrastructuraux pulmonaires ches let rat de l'intoxication oxygénée a la pression atmosphérique. Presse med., 77: 1073 (1969)

29. Payne, J. W., and Patz, A.: Current status of retrolental fibroplasia. Ann. Clin. Res., 11: 205 (1979).

30. Polgar, Antagnoi, W., Ferrigan, L. W., Martin, E. A., and Gregg, W., P.: The effect of chronic exposure to $100 \%$ oxygen in newborn mice. Am. J. . Sci., 252: 580 (1966).

31. Reuckert, R. R., and Mueller, G. C.: Effect of oxygen tension on HeLa cell growth. Cancer Res., 20: 944 (1960).

32. Richards, G. M.: Modifications of the diphenylamine reaction giving increased sensitivity and simplicity in the estimation of DNA. Anal. Biochem., 57: 369 (1974)

33. Smith. F. J. C., Bennett, G. A., Hein, J. W., Thomson, R. M., and Drinker, C. $\mathrm{K} .:$ Morphological changes in the lungs of rats living under compressed air conditions. J. Exp. Med., 56: 79 (1932).

34. Stevens, J. B., and Autor, A. P.: A proposed mechanism for neonatal rat tolerance to normobaric hyperoxia. Fed. Proc. 39: 3138 (1980).

35. Taeusch, H. W., J., Wyszogrodski, I., Wang, N. S., and Avery, M. E.: Pulmonary pressure-volume relationships in premature, fetal, and newborn rabbits. $\mathbf{J}$. Appl. Physiol., 37: 809 (1974).

36. Välimäki, M., Pelliniemi, T., and Niinikoski, J.: Oxygen-induced changes in pulmonary phospholipids in the rat. J. Appl. Physiol., 39: 780 (1975)

37. Weibel, E. W.: Oxygen effects on lung cells. Arch. Intern. Med., 128: 54 (1971)

38. Yam, J., Frank, L., and Roberts, R. J.: Oxygen toxicity: Comparison of lung biochemical responses in neonatal and adult rats. Pediatr. Res., 12: 115 (1978)

39. Yam. J., and Roberts, R. J.: Oxygen-induced lung injury in the newborn piglet. Early Hum. Dev., 41: 411 (1980).

40. The authors wish to thank Mary Jo Kline for her expert technical assistance and Jack Burke for his excellent assistance with the histology.

41. Requests for reprints should be addressed to: R. J. Roberts, M.D., PhD. Department of Pediatrics, BSB 2-530, University of Iowa College of Medicine, University of Iowa, Iowa City, IA 52242 (USA)

42. This research was supported by a grant $(\mathrm{GM} 12675)$ from the General Medical Science Division and a training grant (GM07069) from the United States Public Health Service.

43. Received for publication July 15, 1980.

44. Accepted for publication October $28,1980$. 